\title{
Simple completable contractions of nilpotent Lie algebras
}

\author{
Rutwig Campoamor-Stursberg* \\ Departamento de Geometría y Topología \\ Fac. CC. Matemáticas Univ. Complutense \\ 28040 Madrid ( Spain )
}

\begin{abstract}
We study a certain class of non-maximal rank contractions of the nilpotent Lie algebra $\mathfrak{g}_{m}$ and show that these contractions are completable Lie algebras. As a consequence a family of solvable complete Lie algebras of non-maximal rank is given in arbitrary dimension. .

AMS Math. Subj. Class. 17B10, 17B30.

Keywords : contraction, complete Lie algebra
\end{abstract}

\section{Generalities}

The notion of contraction of a Lie algebra ( also called degeneration by some authors) was originally introduced by physicists ( Segal) as a tool to relate classical and quatum mechanics. Inonu and Wigner used contractions attending to a particularization, namely, that a subalgebra remains fixed through the contraction. This concept, quite restrictive for some purposes, was later generalized by Saletan [13] and Levi-Nahas. The relation between contractions and deformation theory is an important, but not fully exploited question [7]. Orbit closures of Lie algebras, where contractions play a central role, is not an easy problem, and it constitutes an essential tool in the analysis of the componets of the varieties $\mathfrak{L}^{n}$ and $\mathfrak{N}^{n}$.

Let $\mathfrak{L}^{n}$ be the set of complex Lie algebra laws in dimension $n$. We identify each law with its structure constants $C_{i j}^{k}$ on a fixed basis $\left\{X_{i}\right\}$ of $\mathbb{C}^{n}$. The Jacobi identities

$$
\sum_{l=1}^{n} C_{i j}^{k} C_{k l}^{s}+C_{j k}^{l} C_{i l}^{s}+C_{k i}^{l} C_{j l}^{s}=0
$$

for $1 \leq i \leq j<k \leq n, 1 \leq s \leq n$ show that $\mathfrak{L}^{n}$ is an algebraic variety. The nilpotent Lie algebra laws $\mathfrak{N}^{n}$ are a closed subset in $\mathfrak{L}^{n}$. The

*e-mail: rutwig@nfssrv.mat.ucm.es 
linear group $G L(n, \mathbb{C})$ acts on $\mathfrak{L}^{n}$ via changes of basis, i.e., $(g * \mu)(x, y)=$ $g\left(\mu\left(g^{-1}(x), g^{-1}(y)\right)\right)$ for $g \in G L(n, \mathbb{C})$. Let $\mathcal{O}(\mu)$ denote the orbit of the law $\mu$ by this action, consisting of all structures in a single isomorphism class.

Definition. A Lie algebra $\lambda$ is a contraction of a law $\mu$ if $\lambda \in \overline{O(\mu)}$.

Here the topology on the variety is either the metric topology or the Zariski topology. Both topologies lead to the same contractions. A contraction will be denoted by $\mu \longmapsto \lambda$. It follows that the entire orbit $O(\lambda)$ lies in the Zariskiclosure of $O(\mu)$. In particular, the following condition implies $\mu \longmapsto \lambda$ :

$$
\exists g_{t} \in G L(n, \mathbb{C}(t)) \text { such that } \lim _{t \rightarrow 0} g_{t} * \mu=\lambda
$$

Contractions are also transitive, i.e., if $\lambda \longmapsto \mu$ and $\mu \longmapsto \psi$, then $\lambda \longmapsto \psi$. Thus not any existing contraction must be shown directly.

For a Lie algebra $\lambda$ to be a contraction of $\mu$, the following conditions are necessary:

Lemma. Let $\mu \longmapsto \lambda$. Then

1. $\operatorname{dim} \operatorname{Der}(\mu)<\operatorname{dim} \operatorname{Der}(\lambda)$

2. $\operatorname{dim}[\lambda, \lambda] \leq \operatorname{dim}[\mu, \mu]$

3. $\operatorname{dim} Z(\lambda) \geq \operatorname{dim} Z(\mu)$

4. $\operatorname{rank}(\lambda) \geq \operatorname{rank}(\mu)$

where Der $(\mu)$ denotes the algebra of derivations, $Z(\mu)$ the center of the algebra $\mu$ and rank $(\mu)$ is the dimension of a maximal toral subalgebra of $\mu$.

Proofs of the assertions can be found in [5], [7] and [14].

For a nilpotent Lie algebra $\mathfrak{g}$, we denote the ideals of the central descending sequence by $C^{i}(\mathfrak{g})$, i.e., $C^{i}(\mathfrak{g})=\left[\mathfrak{g}, C^{i-1}(\mathfrak{g})\right]$ for $i \geq 1$ and $C^{0}(\mathfrak{g})=\mathfrak{g}$. If the algebra $\mathfrak{g}$ contracts to an algebra $\mathfrak{h}$, it follows from the lemma above that $\operatorname{dim}\left(C^{i}(\mathfrak{g})\right) \geq \operatorname{dim}\left(C^{i}(\mathfrak{g})\right)$. Therefore, if $\mathfrak{g}$ has nilindex $p$, then the nilindex of $\mathfrak{h}$ is $\leq p$.

Although we will not make explicit use of the characteristic sequence, it is convenient to recall this invariant: Consider a complex nilpotent Lie algebra $\mathfrak{g}=$ $\left(\mathbb{C}^{n}, \mu\right)$. For each $X \in \mathbb{C}^{n}$ we denote $c(X)$ the ordered sequence of dimensions of Jordan blocks of the adjoint operator $a d_{\mu}(X)$.

Definition. The characteristic sequence of $\mathfrak{g}$ is an isomorphism invariant $c(\mathfrak{g})$ defined as

$$
c(\mathfrak{g})=\sup _{X \in \mathfrak{g}-C^{1} \mathfrak{g}}\{c(X)\}
$$

where $C^{1} \mathfrak{g}$ denotes the derived subalgebra.

$A$ characteristic sequence is called linear if there exists an integer $n$ such that $c(\mathfrak{g})=(n, 1, . ., 1)$. 


\section{The algebras $\mathfrak{g}_{m}\left(q_{1}, . ., q_{k}\right)$}

In this section we analyze some families of nilpotent Lie algebras for which certain classes of contractions will be determined.

For $m \geq 4$ let $\mathfrak{g}_{m}$ be the Lie algebra whose structural equations are

$$
\begin{aligned}
d \omega_{1} & =d \omega_{2}=0 \\
d \omega_{j} & =\omega_{1} \wedge \omega_{j-1}, 3 \leq j \leq 2 m \\
d \omega_{2 m+1} & =\sum_{j=2}^{m}(-1)^{j} \omega_{j} \wedge \omega_{2 m+1-j}
\end{aligned}
$$

where $\left\{\omega_{1}, . ., \omega_{2 m+1}\right\}$ is a basis of $\left(\mathbb{C}^{2 m+1}\right)^{*}$.

Proposition. For any $m \geq 4$ the Lie algebra $\mathfrak{g}_{m}$ is naturally graded of characteristic sequence $(2 m-1,1,1)$ satisfying the following property

$$
\begin{array}{r}
C_{\mathfrak{g}_{m}}\left(C^{m}\left(\mathfrak{g}_{m}\right)\right) \supset C^{m}\left(\mathfrak{g}_{m}\right) \\
C_{\mathfrak{g}_{m}}\left(C^{m-1}\left(\mathfrak{g}_{m}\right)\right) \not \supset C^{m-1}\left(\mathfrak{g}_{m}\right)
\end{array}
$$

This algebra, which has been analyzed in [3], can be characterized as follows:

Theorem. For $m \geq 4$ any naturally graded, central extension $\mathfrak{g}$ of the filiform model Lie algebra $L_{2 m}$ whose nilindex is $(2 m-1)$ and satisfies

$$
\begin{array}{r}
C_{\mathfrak{g}}\left(C^{m}(\mathfrak{g})\right) \supset C^{m}(\mathfrak{g}) \\
C_{\mathfrak{g}}\left(C^{m-1}(\mathfrak{g})\right) \not \supset C^{m-1}(\mathfrak{g})
\end{array}
$$

is isomorphic to $\mathfrak{g}_{m}$.

Algebras satisfying the preceding "centralizer condition" arise from the analysis of gradations of nilradicals of Borel subalgebras of complex simple Lie algebras, and are defined by a modification of the graded structure of these algebras [3]. In particular, the deformations and extensions of $\mathfrak{g}_{m}$ have been studied in [4].

Let $m \geq 4$. For any sequence $3 \leq q_{1}<q_{2}<\ldots<q_{k} \leq m+1$ let $\mathfrak{g}_{m}\left(q_{1}, ., q_{k}\right)$ be the $(2 m+1)$-dimensional Lie algebra whose Maurer-Cartan equations are:

$$
\begin{aligned}
d \omega_{1} & =d \omega_{2}=0 \\
d \omega_{q_{i}} & =d \omega_{2 m+2-q_{i}}=0,1 \leq i \leq k \\
d \omega_{j} & =\omega_{1} \wedge \omega_{j-1}, 3 \leq j \leq 2 m, j \notin\left\{q_{i}, 2 m+2-q_{i}\right\}_{1 \leq i \leq k} \\
d \omega_{2 m+1} & =\sum_{j=2}^{m}(-1)^{j} \omega_{j} \wedge \omega_{2 m+1-j}
\end{aligned}
$$

where $\left\{\omega_{1}, . ., \omega_{2 m+1}\right\}$ is a basis of $\left(\mathbb{C}^{2 m+1}\right)^{*}$. 
Lemma. For $m \geq 4$ and $k \geq 1$ the Lie algebras $\mathfrak{g}_{m}\left(q_{1}, ., q_{k}\right)$ are nonsplit nilpotent of nonlinear characteristic sequence.

Remark. In general, the algebras $\mathfrak{g}_{m}\left(q_{1}, ., q_{k}\right)$ will not be naturally graded. In particular this happens whenever we have $q, q^{\prime}$ such that $q^{\prime}=1+q$. In fact, the differential form $d \omega_{2 m+1}$ determines the gradation in some sense [3]. These algebras are also interesting for the study of solvable rigid Lie algebras whose nilradical has linear characteristic sequence [2].

Theorem. For any $m \geq 4$ and $k \geq 1$ the Lie algebra $\mathfrak{g}_{m}\left(q_{1}, ., q_{k}\right)$ is a contraction of $\mathfrak{g}$.

Proof. For any $k$-tuple $\left(q_{1}, . ., q_{k}\right)$ let $S\left(m, q_{1}, . ., q_{k}\right)$ be the following linear system

$$
\begin{aligned}
a_{1}+a_{j-1} & =a_{j}, 3 \leq j \leq 2 m, j \notin\left\{q_{i}, 2 m+2-q_{i}\right\}_{1 \leq i \leq k} \\
a_{1}+a_{j-1}-a_{j-1} & =-1, j \in\left\{q_{i}, 2 m+2-q_{i}\right\}_{1 \leq i \leq k} \\
a_{j}+a_{2 m+1-j} & =a_{j+1}+a_{2 m-j}, 2 \leq j \leq m-1
\end{aligned}
$$

and let $S^{\prime}\left(m, q_{1}, . . q_{k}\right)$ be the system given by (1) and (2). We claim that any solution of $S^{\prime}\left(m, q_{1}, . ., q_{k}\right)$ is also a solution of $S\left(m, q_{1}, . ., q_{k}\right)$. In fact, if $j \notin$ $\left\{q_{i}, 2 m+2-q_{i}\right\}_{1 \leq i \leq k}$ then $2 m+2-q_{i} \neq 2 m+1-j$ for all $i$, and therefore we have

$$
a_{j}+a_{2 m+1-j}=a_{j+1}+a_{2 m-j}=a_{1}+a_{j}+a_{2 m-j}
$$

thus

$$
a_{2 m+1-j}=a_{1}+a_{2 m-j}
$$

If $j \in\left\{q_{i}, 2 m+2-q_{i}\right\}_{1 \leq i \leq k}$ then $2 m+2-q_{i}=2 m+1-j$ for some $i \in\{1, . ., k\}$. Then

$$
a_{j}+a_{2 m+1-j}=a_{j+1}+a_{2 m-j}=1+a_{1}+a_{j}+a_{2 m-j}
$$

and simplifying

$$
a_{2 m+1-j}=1+a_{1}+a_{2 m-j}
$$

This shows that the equations (3) are superfluous. Now, the system $S^{\prime}\left(m, q_{1}, . . q_{k}\right)$ clearly has integer solutions, depending on the parameters $a_{2}=N_{1}$ and $a_{3}=N_{2}$. Let $\left(a_{1}, . ., a_{2 m}\right)$ be the solution corresponding to the values $N_{1}=N_{2}=1$ and $T_{2 m+1}(\mathbb{C}(t))$ denote the Borel subgroup of $G L(2 m+1, \mathbb{C})$ consisting of lower triangular matrices. Define $f_{\left(q_{1}, . ., q_{k}\right), t} \in T_{2 m+1}(\mathbb{C}(t))$ by

$$
\left\{\begin{array}{l}
f_{\left(q_{1}, . ., q_{k}\right), t}\left(X_{i}\right)=t^{a_{i}} X_{i}, i \neq 2 m+1 \\
f_{\left(q_{1}, . ., q_{k}\right), t}\left(X_{2 m+1}\right)=t^{1+a_{2 m-1}}
\end{array}\right.
$$


and consider the Lie algebra $f_{\left(q_{1}, . ., q_{k}\right), t}^{-1} * \mu$, where $\mu$ is the Lie algebra law associated to $\mathfrak{g}_{m}$. Then the structural equations of $f_{\left(q_{1}, . ., q_{k}\right), t}^{-1} * \mu$ are given by

$$
\begin{aligned}
d \omega_{1} & =d \omega_{2}=0 \\
d \omega_{j} & =t^{a_{1}+a_{j-1}-a_{j}} \omega_{1} \wedge \omega_{j-1}, j \in\left\{q_{i}, 2 m+2-q_{i}\right\}_{1 \leq i \leq k} \\
d \omega_{j} & =\omega_{1} \wedge \omega_{j-1}, 3 \leq j \leq 2 m, j \notin\left\{q_{i}, 2 m+2-q_{i}\right\}_{1 \leq i \leq k} \\
d \omega_{2 m+1} & =\sum_{j=2}^{m}(-1)^{j} \omega_{j} \wedge \omega_{2 m+1-j}
\end{aligned}
$$

Now, as $a_{1}+a_{j-1}-a_{j}=-1$ for $j \in\left\{q_{i}, 2 m+2-q_{i}\right\}_{1 \leq i \leq k}$ by the system $S^{\prime}\left(m, q_{1}, . ., q_{k}\right)$ ( therefore by $\left.S\left(m, q_{1}, . ., q_{k}\right)\right)$, it follows easily that

$$
\lim _{t \rightarrow \infty} f_{\left(q_{1}, . ., q_{k}\right), t}^{-1} * \mu=\mu\left(q_{1}, . ., q_{k}\right)
$$

where $\mu\left(q_{1}, . ., q_{k}\right)$ is the law associated to $\mathfrak{g}_{m}\left(q_{1}, . ., q_{k}\right)$.

Corollary. For $m \geq 4$

$$
\mu\left(q_{1}, . ., q_{k}\right) \in O\left(\mu\left(q_{1}^{\prime}, . ., q_{k}^{\prime}\right)\right)
$$

if and only if $\left\{q_{1}, . ., q_{k}\right\}=\left\{q_{1}^{\prime}, . ., q_{k}^{\prime}\right\}$.

Corollary. For $m \geq 4$ the algebras $\mathfrak{g}_{m}\left(q_{1}, . ., q_{k}\right)$ (included $\mathfrak{g}$ ) are nontrivial deformations of the algebra $\mathfrak{h}_{m-1} \oplus \mathbb{C}^{2}$, where $\mathfrak{h}_{m-1}$ is the $(2 m-1)$-dimensional Heisenberg Lie algebra.

Proof. Let $f_{t} \in T_{2 m+1}(\mathbb{C}(t))$ be defined by

$$
f_{t}\left(X_{i}\right)=t^{a_{i}} X_{i}, 1 \leq i \leq 2 m+1
$$

where the $a_{i}$ satisfy the system $S^{\prime}\left(m, q_{1}, . ., q_{m}\right)$ and the additional equation

$$
a_{1}+a_{2 m-1}=a_{2 m}-1
$$

Then $\lim _{t \rightarrow \infty} f_{t}^{-1} * \mu \in O\left(\mathfrak{h}_{m-1} \oplus \mathbb{C}^{2}\right)$.

A similar reasoning shows that $\mathfrak{g}_{m}\left(q_{1}, . ., q_{k}\right) \longmapsto \mathfrak{h}_{m-1} \oplus \mathbb{C}^{2}$. The result follows from the fact that a contraction defines a nontrivial deformation [7].

\section{Applications to complete Lie algebras}

Recall that a Lie algebra $\mathfrak{g}$ is called complete if it is centerless and any derivation is inner [8]. I recent years, a general theory of complete solvable Lie algebras whose nilradical is of maximal rank has been developed ( [9], [10], [12]), and the existence of non-maximal rank algebras has been pointed out. In this section we will see how to obtain families of non-maximal rank completable Lie algebras considering the contractions above. 
Let $\mathfrak{g}$ be a nilpotent Lie algebra and $\operatorname{Der}(\mathfrak{g})$ its Lie algebra of derivations. A torus $\mathfrak{t}$ over $\mathfrak{g}$ is an abelian subalgebra of $\operatorname{Der}(\mathfrak{g})$ consisting of semisimple derivations. The torus $\mathfrak{t}$ induces a natural represntation on $\mathfrak{g}$ such that

$$
\mathfrak{g}=\sum_{\alpha \in \mathfrak{t}^{*}} \mathfrak{g}_{\alpha}
$$

where $\mathfrak{t}^{*}=\operatorname{Hom}(\mathfrak{t}, \mathbb{C})$ and $\mathfrak{g}_{a}=\{X \in \mathfrak{g} \mid[t, X]=\alpha(t) X, \forall t \in \mathfrak{t}\}$. If $\mathfrak{t}$ is maximal for the inclusion relation, by the conjugation theorems of Morozov, its common dimension is a numerical invariant called the rank of $\mathfrak{g}$, denoted $\operatorname{rank}(\mathfrak{g})$. An algebra is called of maximal rank if $\operatorname{rank}(\mathfrak{g})=b_{1}=\operatorname{dim} H^{1}(\mathfrak{g}, \mathbb{C})$. Following Favre [6], a weight system of $\mathfrak{g}$ is given by

$$
P \mathfrak{g}(\mathfrak{t})=\left\{(\alpha, d \alpha) \mid \alpha \in t^{*} \text { such that } \mathfrak{g}_{\alpha} \neq 0, d a=\operatorname{dim} \mathfrak{g}_{\alpha}\right\}
$$

We also recall the folowing results:

Proposition. Let $\mathfrak{g}$ be of maximal rank and $\mathfrak{t}$ a maximal torus. Then the semidirect product $\mathfrak{t} \oplus \mathfrak{g}$ is complete solvable.

Definition. The algebra $\mathfrak{g}$ is called completable if $\mathfrak{t} \oplus \mathfrak{g}$ is complete for a maximal torus $\mathfrak{t}$, and simple completable if it is not the direct sum of nontrivial completable Lie algebras.

The main result we will use is a slight modification of the next

Theorem. Let $\mathfrak{g}$ be a Lie algebra and $\mathfrak{h}$ a Cartan subalgebra. Assume that following conditions are satisfied:

1. $\mathfrak{h}$ is abelian

2. $\mathfrak{g}=\sum_{\alpha \in \Delta} \mathfrak{g}_{\alpha}$ with $\Delta \subset \mathfrak{h}^{*}-\{0\}$

3. there is a basis $\left\{\alpha_{1}, . ., \alpha_{l}\right\}$ of $\mathfrak{h}^{*} n \Delta$ such that $\operatorname{dim} \mathfrak{g}_{ \pm \alpha_{j}} \leq 1$ for $1 \leq j \leq l$ and $\left[\mathfrak{g}_{\alpha_{j}}, \mathfrak{g}_{-\alpha_{j}}\right] \neq 0$ if $-\alpha_{j} \in \Delta$

4. $\mathfrak{h}$ and $\left\{\mathfrak{g}_{ \pm \alpha_{j}}, 1 \leq j \leq l\right\}$ generate $\mathfrak{g}$

Then $\mathfrak{g}$ is a complete Lie algebra.

The last conditions can be replaced by by more general statements [11]

3 ' there is a enerating system $\left\{\alpha_{1}, . ., \alpha_{l}\right\}$ of $\mathfrak{h}^{*}$ in $\Delta$ such that $\operatorname{dim} \mathfrak{g}_{\alpha_{j}}=1$ for all $j$ and $\mathfrak{h}, \mathfrak{g}_{\alpha_{1}}, . ., \mathfrak{g}_{\alpha_{l}}$ genrate $\mathfrak{g}$.

4' Let $0 \neq x_{j} \in \mathfrak{g}_{\alpha_{j}}$ and a basis $\left\{\alpha_{1}, . ., \alpha_{r}\right\}$ of $\mathfrak{h}^{*}$. For $r+1 \leq s \leq l$,

$$
\alpha_{s}=\sum_{i=1}^{t} k_{i s} \alpha_{j_{i}}-\sum_{i=1+t}^{r} k_{i s} \alpha_{j_{i}}
$$


where $k_{i s} \in \mathbb{N} \cup\{0\},\left(j_{1}, . ., j_{r}\right)$ is a permutation of $(1, . ., r)$, and there is a formula

$$
\begin{gathered}
{[\underbrace{x_{j_{1}}, \ldots, x_{j_{1}}}_{k_{1 s}}, . \underbrace{x_{j_{t}}, . ., x_{j_{t}}}_{k_{t s}}, \ldots, x_{k_{m}}]} \\
=[\underbrace{x_{j_{t}+1}, . ., x_{j_{t}+1}}_{k_{t+1 s}}, . . \underbrace{x_{j_{r}}, \ldots, x_{j_{r}}}_{k_{r s}}, x_{s}, x_{k_{1}} \ldots, x_{k_{m}}]
\end{gathered}
$$

without regard to the order or the way of bracketing, where $m \neq 0$ if $t=r$.

Theorem [11]. Let $\mathfrak{g}$ be a Lie algebra satisfying conditions $(1),(2),\left(3^{\prime}\right)$ and $\left(4^{\prime}\right)$. Then $\mathfrak{g}$ is a complete Lie algebra.

We obtain one more consecuence of the theorem in the preceding section

Corollary. For $m \geq 4$ and $k \geq 1$

$$
\operatorname{rank}\left(\mathfrak{g}_{m}\left(q_{1}, . ., q_{k}\right)\right)>2
$$

Proof. The fact follows from the linear system $\left(S\left(\mathfrak{g}_{m}\left(q_{1}, . ., q_{k}\right)\right)\right)$ associated to the algebras [1] and the fact that $b_{1}\left(\mathfrak{g}_{m}\left(q_{1}, . ., q_{k}\right)\right)>b_{1}\left(\mathfrak{g}_{m}\right)=2$, where $b_{1}(\mathfrak{g})=\operatorname{dim} H^{1}(\mathfrak{g}, \mathbb{C})$.

Proposition. Let $m \geq 4$ and $k \geq 1$ a weight system of $\mathfrak{g}_{m}\left(q_{1}, . ., q_{k}\right)$ is given by

$$
P \mathfrak{g}_{m}\left(\mathfrak{t}_{m}\right)=\left\{\left(\alpha_{i}, d \alpha_{i}\right)_{1 \leq i \leq 2 m+1}\right\}
$$

where $\operatorname{dim} \mathfrak{g}_{\alpha_{i}}=1$ for all $i$ and the weights $\left\{\alpha_{1}, . ., \alpha_{2 m+1}\right\}$ satisfy the following linear system

$$
\begin{aligned}
\alpha_{1}+\alpha_{j-1} & =a_{j}, & & 3 \leq j \leq 2 m, j \notin\left\{q_{i}, 2 m+2-q_{i}\right\}_{1 \leq i \leq k} \\
a_{2 m-t}+-\alpha_{t+1}-\alpha_{m} & =\alpha_{m+1}, & & 1 \leq t \leq m-2
\end{aligned}
$$

In particular, $\operatorname{rank}\left(\mathfrak{g}_{m}\left(q_{1}, . ., q_{k}\right)\right) \leq m+1$.

Proof. The system $\left(S_{1}\right)$ coincides with the linear system $S\left(\mathfrak{g}_{m}\left(q_{1}, . ., q_{k}\right)\right)$ associated to the nilpotent Lie algebra $\mathfrak{g}_{m}\left(q_{1}, . ., q_{k}\right)$, thus the $\alpha_{i}$ correspond to eigenvalues of semisimple derivations [1]. Therefore we can isolate $\alpha_{j_{1}}, . ., \alpha_{j_{s}}$ $\left(1 \leq j_{1}<j_{2} . .<j_{s}<2+2 k\right)$ such that for any $j \in\{1,,, 2 m+1\}-\left\{j_{1}, . ., j_{s}\right\}$ we have

$$
\alpha_{j}=\sum_{t=1}^{s} a_{j}^{t} \alpha_{j_{t}}, a_{j}^{t} \in \mathbb{C}
$$

Now, using the standard techniques [1] it is routine to verify that for $1 \leq t \leq s$ the derivations $f_{j_{t}} \in \operatorname{Der}\left(\mathfrak{g}_{m}\left(q_{1}, . ., q_{k}\right)\right)$ given by

$$
f_{j_{t}}\left(X_{i}\right)=a_{i}^{t} X_{i}, 1 \leq i \leq 2 m+1
$$


define a maximal torus $\mathfrak{t}_{m}\left(q_{1}, . ., q_{k}\right)$ of $\mathfrak{g}_{m}\left(q_{1}, . ., q_{k}\right)$. Thus the weight system is given as above.

Clearly any weight space $\mathfrak{g}_{\alpha_{j}}$ is at most one dimensional. For the last assertion, observe that for $k=m+1$ the system $\left(S_{1}\right)$ is

$$
\begin{aligned}
\alpha_{1}+\alpha_{2 m-1} & =\alpha_{2 m} \\
\alpha_{t}+\alpha_{2 m+1-t} & =\alpha_{m}+\alpha_{m+1}, 2 \leq t \leq m-1
\end{aligned}
$$

and that the rank is the maximal possible, namely $m+1$.

Theorem. For $m \geq 4$ and $k \geq 1$ the semidrect products

$$
\mathfrak{r}_{m}\left(q_{1}, . ., q_{k}\right)=\mathfrak{t}_{m}\left(q_{1}, . ., q_{k}\right) \oplus \mathfrak{g}_{m}\left(q_{1}, . ., q_{k}\right)
$$

are solvable and complete.

Proof. It is easy to see that for any $f \in \operatorname{Der}\left(\mathfrak{r}_{m}\left(q_{1}, . ., q_{k}\right)\right)$ we have

$$
f\left(\mathfrak{t}_{m}\left(q_{1}, . ., q_{k}\right)\right) \subset \mathfrak{g}_{m}\left(q_{1}, . ., q_{k}\right)
$$

This is a direct consequence of the particular action of $\mathfrak{t}_{m}\left(q_{1}, . ., q_{k}\right)$ over $\mathfrak{g}_{m}\left(q_{1}, . ., q_{k}\right)$. If $\mathfrak{t}_{m}\left(q_{1}, . ., q_{k}\right)=\left\{h_{1}, . ., h_{s}\right\}$, then for any $1 \leq t \leq s$ there exists a permutation $\sigma \in \mathcal{S}_{s}$ such that $f_{j_{t}}=a d\left(h_{t}\right)$. The nilradical is clearly generated by $\left\{X_{1}, X_{2}, X_{q_{i}}, X_{2 m+2-q_{i}}\right\}_{1 \leq i \leq k}$, where $\left\{X_{1}, . ., X_{2 m+1}\right\}$ is a dual basis to $\left\{\omega_{1}, . ., \omega_{2 m+1}\right\}$. It follows that the algebra $\mathfrak{r}_{m}\left(q_{1}, . ., q_{k}\right)$ satisfies the conditions of the preceding theorem, so that it is complete.

Lemma. If $k=1$ and $q_{1} \neq m+1$ or $k \geq 2$, the $\mathfrak{g}_{m}\left(q_{1}, . ., q_{k}\right)$ is not of maximal rank.

Proof. The proof is an immediate consequence of the weight system. For $k=1$ and $q_{1}=m+1$ we have $\operatorname{rank}\left(S_{1}\right)=b_{1}=3$. Observe that this is the only case where $q_{i}=2 m+2-q_{i}$.

Corollary. For any odd dimension $n \geq 9$ there exist completable Lie algebras of non-maximal rank.

Thus the contractions of the algebra $\mathfrak{g}_{m}$ (it is itself completable since it is of maximal rank) are completable of non-maximal rank, up to an exception. The families above expand the examples obtained in [10],[12] for non-maximal rank. In fact, since the algebras $\mathfrak{g}_{m}\left(q_{1}, . ., q_{k}\right)$ are nonsplit, we obtain even more:

Corollary. For $m \geq 4$ and $k \geq 1$ the algebras $\mathfrak{g}_{m}\left(q_{1}, . ., q_{k}\right)$ are simple completable. 


\section{References}

[1] J. M. Ancochea, M. Goze. Le rang du système lineaire des racines d'une algèbre de Lie resoluble complexe. Comm. Algebra 20 (1992), 875-887.

[2] J. M. Ancochea, R. Campoamor. On Lie algebras whose nilradical is (n-p)filiform. Comm. Algebra 29 (2001), 427-450.

[3] J. M. Ancochea, R. Campoamor. On certain families of naturally graded Lie algebras. J. Pure Appl. Algebra, to appear.

[4] J. M. Ancochea, R. Campoamor. Nonfiliform characteristically nilpotent and complete Lie algebras. Algebra. Colloq., to appear.

[5] D. Burde. Degenerations of nilpotent Lie algebras. J. Lie Theory 9 (1999), 193-202.

[6] G. Favre Système des poids sur une algèbre de Lie nilpotente. Manuscripta Math.9 (1973), 53-90.

[7] A. Fialowski, J. O'Halloran. A comparison of deformations and orbit closure. Comm. Algebra (18) (1990), 4121-4140.

[8] G. F. Leger. Derivations of Lie algebras III. Duke Math. J. 30 (1963), 637-645.

[9] D. J. Meng, L. S. Zhu. Solvable complete Lie algebras I. Comm. Algebra 24 (1996), 4181-4197.

[10] D. J. Meng, L. S. Zhu. Solvable complete Lie algebras II. Algebra Colloq. 5 (1998), 289-296.

[11] D. J. Meng. Complete Lie algebras and Heisenberg Lie algebras. Comm. Algebra 22 (1994), 5509-5524.

[12] D. J. Meng. The complete Lie algebras with abelian nilpotent radical. Acta Math. Sinica 34 (1991), 191-202.

[13] E. J. Saletan. Contractions of Lie groups. J. Math. Phys. 2 (1961), 1-21.

[14] C. Seeley. Degenerations of central quotients. Arch. Math. 56 (1991), 236241. 